\title{
Testing Dark Decays of Baryons in Neutron Stars
}

\author{
Gordon Baym, ${ }^{1}$ D. H. Beck, ${ }^{1}$ Peter Geltenbort, ${ }^{2}$ and Jessie Shelton ${ }^{1}$ \\ ${ }^{1}$ Department of Physics, University of Illinois, 1110 West Green Street, Urbana, Illinois 61801, USA \\ ${ }^{2}$ Institut Max von Laue Paul Langevin, 71 avenue des Martyrs, F-38042 Grenoble Cedex 9, France
}

(Received 23 February 2018; revised manuscript received 27 June 2018; published 6 August 2018)

\begin{abstract}
The observation of neutron stars with masses greater than one solar mass places severe demands on any exotic neutron decay mode that could explain the discrepancy between beam and bottle measurements of the neutron lifetime. If the neutron can decay to a stable, feebly interacting dark fermion, the maximum possible mass of a neutron star is $0.7 M_{\odot}$, while all well-measured neutron star masses exceed one $M_{\odot}$. The existence of $2 M_{\odot}$ neutron stars further indicates that any explanation beyond the standard model for the neutron lifetime puzzle requires dark matter to be part of a multiparticle dark sector with highly constrained interactions. Beyond the neutron lifetime puzzle, our results indicate that neutron stars provide unique and useful probes of $\mathrm{GeV}$-scale dark sectors coupled to the standard model via baryon-number-violating interactions.
\end{abstract}

DOI: 10.1103/PhysRevLett.121.061801

The neutron lifetime anomaly, the discrepancy in the beam [1,2] vs bottle [3-9] measurements of the lifetime of the neutron, is a long-standing puzzle [10,11]. Briefly, the bottle technique, an inclusive measurement of the neutron lifetime, yields $\tau_{\text {bottle }}=879.6 \pm 0.6 \mathrm{~s}$, which is discrepant at the $4 \sigma$ level with the exclusive measurement of the neutron lifetime via beam experiments, $\tau_{\text {beam }}=888.0 \pm$ $2.0 \mathrm{~s}$ [12]. In a recent Letter, Fornal and Grinstein [13] made the intriguing suggestion that new decay channels of the neutron $n$, in particular,

$$
n \rightarrow \chi+\gamma, \quad n \rightarrow \chi+e^{+} e^{-}, \quad n \rightarrow \chi+\phi,
$$

where $\chi$ is a dark matter fermion, $\phi$ is a dark matter boson, and $\gamma$ is a photon, could explain the shorter lifetime in the bottle experiments. The amplitude for these processes must be sufficiently large to allow a rate of $\Gamma \sim 10^{-5} \mathrm{~s}^{-1}$ to explain the bottle-beam anomaly. This resolution to the neutron decay puzzle faces a number of challenges. The $n \rightarrow \chi+\gamma$ decay mode has been tested in a recent experiment [14] that excluded all branching ratios that could account for the lifetime anomaly. In addition, Ref. [15] argues that recent measurements of the axial renormalization constant $g_{A}$ likely point to a shorter standard model (SM) lifetime of the neutron, more in line with the bottle results.

With this suggestion in mind, we show that neutron stars are powerful laboratories to test proposed dark decays of

Published by the American Physical Society under the terms of the Creative Commons Attribution 4.0 International license. Further distribution of this work must maintain attribution to the author(s) and the published article's title, journal citation, and DOI. Funded by SCOAP . baryons. The conversion of baryons to dark fermions through processes of the form in Eq. (1) lead, in the absence of strong self-interactions of the dark fermions, $\chi$, to a maximum neutron star mass much smaller than observed masses. Thus, the existence of neutron stars with masses up to $2 M_{\odot}$ [16-18] allows us to draw broad and generic conclusions about the type of baryon-numberviolating dark interactions of the neutron required for a beyond the standard model (BSM) explanation of the neutron lifetime puzzle.

The processes of Eq. (1) would convert a fraction of the neutrons present into $\chi$ 's during the formation of a neutron star. The $\chi$ 's would sit in the gravitational potential well of the neutron star, in thermodynamic equilibrium with the normal neutron star matter, and form a noninteracting Fermi gas, similar to a noninteracting neutron gas. (The $10^{5} \mathrm{~s}$ timescale for $n \rightarrow \chi+Y$ needed to resolve the neutron lifetime puzzle is very short compared to inferred neutron star ages, which range from hundreds to billions of years [19].) The basic physics is that, except near nuclear matter density, the interactions of neutrons with the neutron star medium are effectively repulsive, and thus the conversion of a neutron into a weakly interacting dark matter particle is generally highly energetically favored. Figure 1 shows the baryon chemical potential $\mu_{b}$ vs the baryon density $n_{b}$, in units of $n_{0}$, the nuclear matter saturation density, $\simeq 0.16 \mathrm{fm}^{-3}$, for the modern quark-hadron crossover [QHC18 $(0.8,1.5)]$ neutron star matter equation of state [20], for the "stiffer" Akmal-Pandharipande-Ravenhall (APR) equation of state [21], and for free neutrons. The QHC18 equations of state take quark degrees of freedom in the interior into account consistently and allow $2 M_{\odot}$ neutron stars. They are in striking agreement with the equation of state constraints deduced by LIGO from the 


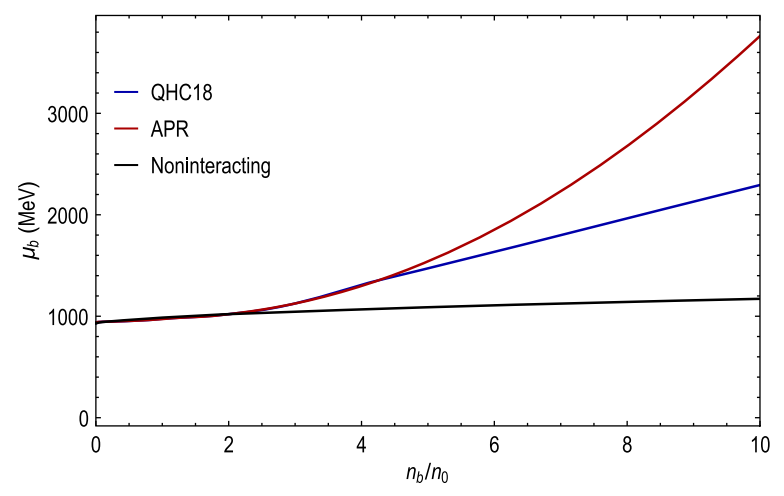

FIG. 1. Baryon (or neutron) chemical potential (including rest mass) in $\mathrm{MeV}$ vs the baryon density in units of nuclear matter density, $n_{0}=0.16 \mathrm{fm}^{-3}$, for the quark-hadron crossover equation of state, QHC18(0.8,1.5) [20], for the stiffer APR equation of state [21], and for noninteracting (free) neutrons. This figure shows how much more expensive it would be for a baryon to remain at high densities instead of turning into a weakly interacting dark matter particle with $m_{\chi}<\mu_{b}$.

recent binary neutron star merger [22]. The ingredients of these equations of state are effectively: (i) the APR equation of state for nuclear matter in beta equilibrium, up to a baryon density of $\simeq 2 n_{0}$; (ii) above a baryon density of $\sim 5 n_{0}$, a quark matter equation of state with a repulsive contact interaction between the quarks with coupling constant $g_{v}$, equal here to $0.8 G$, and an effective BCS pairing interaction between quarks with coupling constant $H$, equal here to $1.5 G$, where $G$ is the Nambu-Jona-Lasinio quark-quark coupling constant; and (iii) between the two extremes, a smooth interpolation of $P$ vs $\mu_{b}$. For a given $n_{b}$, the conversion of neutrons to free fermions of equal mass would generally gain of the order of hundreds of $\mathrm{MeV}$ per neutron.

The interactions (1) are phrased in terms of the neutron instead of the quarks comprising the neutron. Thus, to describe the effects of these interactions on neutron stars, it is simplest to use the language of neutron degrees of freedom, although the calculations we present are valid for more general baryon and quark degrees of freedom. We calculate neutron star models in the presence of a generic interaction $n \rightarrow \chi+Y$, where $Y$ is a possibly multiparticle final state with zero net chemical potential $\mu_{Y}=0$. Such interactions include the highly pertinent SM final states $Y=\gamma, e^{+} e^{-}$as well as a broad range of BSM possibilities such as a dark photon. We assume for simplicity that the $\chi$ 's have spin $1 / 2$.

In a neutron star with noninteracting $\chi$ 's, a sea of neutrons, with Fermi momentum $k_{b}$ and density $n_{b} \equiv$ $k_{b}^{3} / 3 \pi^{2}$, would be in equilibrium with a sea of $\chi$ 's with Fermi momentum $k_{\chi}$, density $n_{\chi}=k_{\chi}^{3} / 3 \pi^{2}$, and chemical potential $\mu_{\chi}^{2}=\sqrt{m_{\chi}^{2}+k_{\chi}^{2}}$. In equilibrium, the $\chi$ 's must have the same chemical potential $\mu_{b}$ as that of the baryons. To calculate the relative population of baryons and $\chi$, we

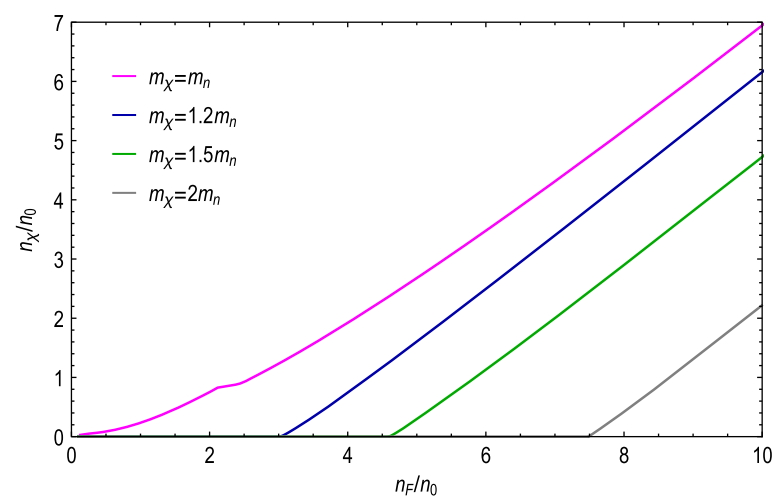

FIG. 2. Number density of dark matter fermions $\chi$ in chemical equilibrium as a function of the total number of fermions, $n_{F}=n_{b}+n_{\chi}$, in units of nuclear matter density $n_{0}$, for the QHC18 neutron star equation of state and for different dark matter fermion masses: $m_{\chi}=m_{n}, m_{\chi}=1.2 m_{n}, m_{\chi}=1.5 m_{n}$, and $m_{\chi}=2 m_{n}$. The small flattening in the $m_{\chi}=m_{n}$ curve reflects the onset of pion condensation in the APR equation of state [21].

write $k_{\chi}=y k_{b}$, where $y$ is itself a function of $k_{b}$. Although we use relativistic kinematics in the numerical results presented below, we provide here the nonrelativistic limits to illustrate the physics most simply. Nonrelativistically, chemical equilibrium leads to

$$
y^{2}=\frac{m_{\chi}\left(\mu_{b}-m_{\chi}\right)}{m_{n}\left(\mu_{n}^{0}-m_{n}\right)}, \quad \mu_{b} \geq m_{\chi}
$$

where $\mu_{n}^{0}$ is the chemical potential of a free neutron gas at density $k_{b}^{3} / 3 \pi^{2}$. We take for simplicity $m_{\chi}=m_{n}$ in addressing the Fornal and Grinstein proposition. Given that $\mu_{b}>m_{n}$ at high densities, we also show results for a range of $m_{\chi}>m_{n}$.

The total density of fermions is $n_{F}=n_{b}+n_{\chi}=$ $n_{b}\left(1+y^{3}\right)$. For $m_{\chi}=m_{n}$, we find that, at total fermion density $n_{F} \simeq n_{0}$ (nuclear matter saturation density), about $40 \%$ of the fermions are $\chi$ 's, while at $n_{F} \simeq 4 n_{0}$ the number of $\chi$ and normal baryons are approximately equal, and, at $n_{F}=10 n_{0}, \sim 70 \%$ of the fermions are $\chi$ 's (see Fig. 2). If the baryon chemical potential is below $m_{\chi}$, no $\chi$ can be present and $y \equiv 0$.

The $\chi$ 's contribute only their rest mass and kinetic energy to the total energy density $\epsilon$ of the matter; again nonrelativistically for illustration

$$
\epsilon=\epsilon_{b}\left(n_{b}\right)+m_{\chi} n_{b} y^{3}+\frac{k_{b}^{5}}{10 \pi^{2} m_{\chi}} y^{5},
$$

while the total pressure is

$$
P=P_{b}\left(n_{b}\right)+\frac{k_{b}^{5}}{15 \pi^{2} m_{\chi}} y^{5},
$$




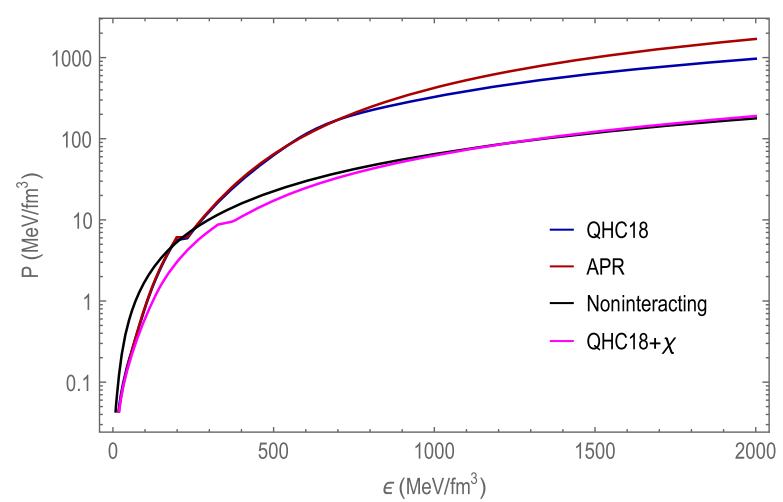

FIG. 3. The equation of state for baryons coupled to $\chi$ 's, with $m_{\chi}=m_{n}$, and for the QHC18(0.8,1.5), APR, and free neutron equations of state. As explained in the text, at the resolution of this graph, the corresponding curves for baryons with the QHC18 and APR equations of state in equilibrium with $\chi$ 's are indistinguishable. Even though the APR equation of state is stiffer at higher densities than QHC18, in chemical equilibrium the core of the star would contain primarily dark fermions in either case. At nuclear matter density, $\epsilon \simeq 150 \mathrm{MeV} / \mathrm{fm}^{3}$.

where $\epsilon_{b}$ is the energy density and $P_{b}$ the pressure of normal matter. The $\chi$ 's increase the energy density more than the pressure and, thus, at high densities soften the equation of state and lower the maximum neutron star mass.

Figure 3 shows the pressure $P(\epsilon)$ calculated numerically for baryons and $\chi$ 's in chemical equilibrium, for normal matter described by both the QHC18 and APR equations of state, as well as for these two equations of state without $\chi$, and for free neutrons. The pressure of the equilibrium baryon- $\chi$ mixture does not depend noticeably on the baryon equation of state here. At high densities, where the pressure is lowered to essentially that of a free neutron gas, the matter is dominated by $\chi$ 's, while, at densities below $2 n_{0}$, the QHC18 and APR equations of state are identical by construction. We show in Fig. 4 the dependence of the total pressure of the coupled system for different $m_{\chi}$; the pressure follows the normal equation of state up to energy densities where $\chi$ 's are first allowed kinematically and then flattens.

The resulting neutron star masses, found by integrating the Tolman-Oppenheimer-Volkov equation [23,24], are shown in Fig. 5 as a function of the central energy density for QHC18 as well as for APR in equilibrium with $\chi$ 's for $m_{\chi}=m_{n}$ (again, the results are indistinguishable at the resolution of the figure), for APR and QHC18 alone, and for free neutrons. The maximum neutron star mass for the coupled matter is reduced from $\sim 2 M_{\odot}$ to $\sim 0.7 M_{\odot}$, even below that for free neutrons; this reduction is a consequence of the QHC18 equation of state at low densities being softer than that of free neutrons (Fig. 3). In Fig. 6, we show, for QHC18 coupled to $\chi$ 's, neutron star masses for a range of $m_{\chi}$; in Fig. 7, we show the mass-radius relations for the

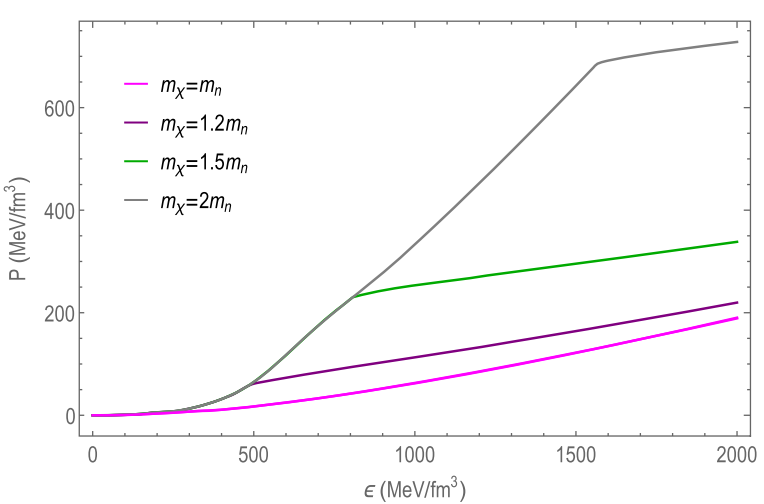

FIG. 4. The QHC18(0.8,1.5) equation of state in chemical equilibrium with dark matter fermions of different masses: $m_{\chi}=m_{n}, m_{\chi}=1.2 m_{n}, m_{\chi}=1.5 m_{n}$, and $m_{\chi}=2 m_{n}$. One sees here the dramatic softening induced by coupling to $\chi$ 's. For larger $m_{\chi}$, the onset of the softening is pushed to higher $\epsilon$, where it becomes energetically favorable for a baryon to be converted to a $\chi$.

same range of $m_{\chi}$. As $m_{\chi}$ increases to $2 m_{n}$, the impact on the neutron star composition is negligible: There exist relatively few $\chi$ 's and only at high densities. We conclude that the assumed coupling of baryons to noninteracting dark matter lowers the maximum neutron star mass to well below that observed, and thus the proposed exotic neutron decay mode is physically untenable unless the dark matter equation of state satisfies very demanding conditions, which we now discuss.

In order to increase the mass of neutron stars with normal matter coupled to dark matter, the dark matter fermions would not only have to be strongly interacting among

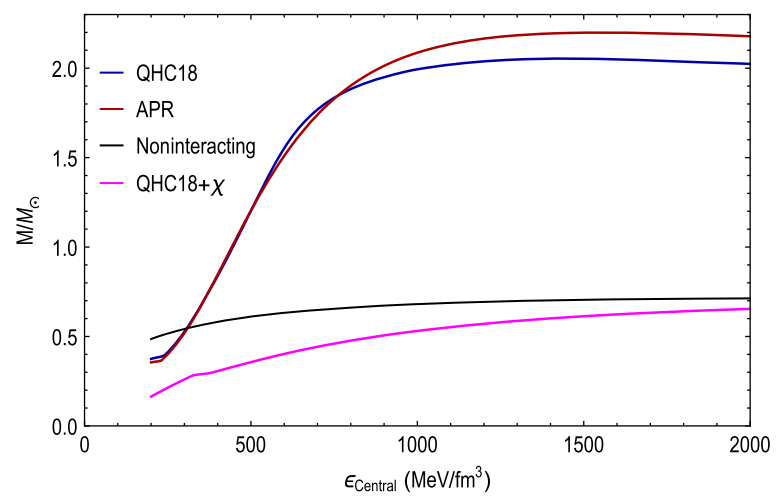

FIG. 5. Neutron star masses vs central density for baryons with the $\mathrm{QHC} 18(0.8,1.5)$ equation of state in chemical equilibrium with $\chi$ 's having $m_{\chi}=m_{n}$ and for the QHC18(0.8,1.5), APR, and free neutron equations of state. This figure shows how coupling of baryons to weakly interacting dark matter precludes an explanation of the existence of neutron stars from $1 M_{\odot}$ to $2 M_{\odot}$. At the resolution of this graph, the corresponding curves for baryons with the QHC18 and APR equations of state in equilibrium with $\chi$ 's are indistinguishable. 


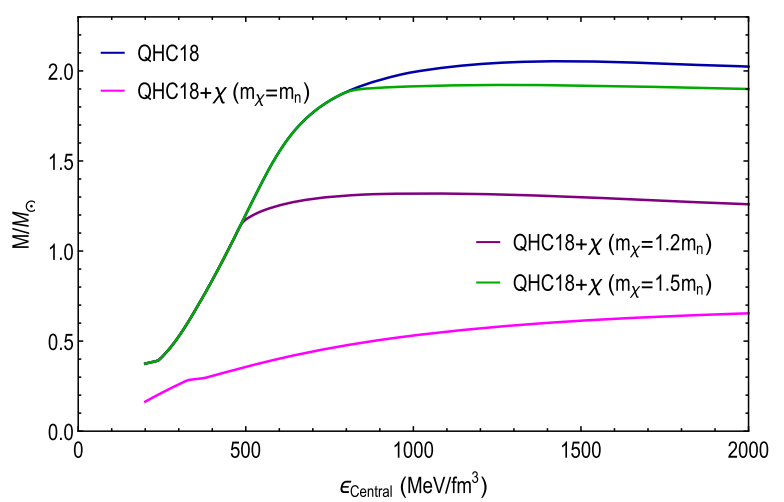

FIG. 6. Neutron star mass as a function of the central energy density with baryons in chemical equilibrium with dark matter fermions for different masses: $m_{\chi}=m_{n}, m_{\chi}=1.2 m_{n}$, and $m_{\chi}=1.5 m_{n}$. At larger $m_{\chi}$, the conversion of baryons to dark matter is kinematically forbidden at lower densities, as shown in Fig. 2. Therefore, the neutron star mass shown here is independent of the dark matter mass for a sufficiently low central density. For $m_{\chi}=2 m_{n}$, the neutron star mass is essentially unaffected by the small number of $\chi$ 's present for this range of central densities.

themselves, but the self-interactions would be strongly constrained. Sizable short-range dark matter selfinteractions are not impossible; the most stringent astrophysical constraint comes from the Bullet Cluster, which allows cross sections $\sigma_{\chi} \lesssim(66 / \mathrm{GeV})^{2} \sim 10^{-24} \mathrm{~cm}^{2}$-on the scale of low-energy baryonic cross sections-for a particle with the mass of a neutron [25]. As a first scenario, one might imagine that dark matter fermions exactly mirror normal matter. Then the energy density and pressure in a neutron star in equilibrium with dark matter, with total fermion density $2 n_{F}$, would be just twice that of the normal matter alone at density $n_{F}$, leading to a reduction in the maximum neutron star mass by $\sqrt{2}$ (see Appendix A of

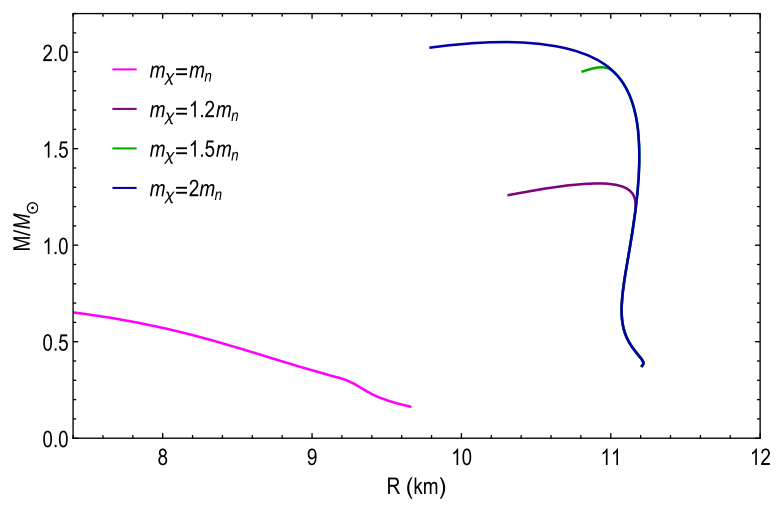

FIG. 7. Neutron star mass as a function of the neutron star radius for baryons with the QHC18 equation of state in chemical equilibrium with $\chi$ 's of mass $m_{\chi}=m_{n}, m_{\chi}=1.2 m_{n}$, and $m_{\chi}=1.5 m_{n}$. Again, the QHC18 curves with no dark matter and for $m_{\chi}=2 m_{n}$ are essentially indistinguishable for this range of central energy densities.
Ref. [20]), that is, a maximum mass of the order of $1.4 M_{\odot}$, below observations. A second possibility could be that the dark matter has sufficiently repulsive interactions to overcome the softening of the equation of state due to adding a second species, thus accommodating $2 M_{\odot}$ neutron stars. Such a scenario makes very specific demands on the dark matter self-interaction strength as a function of the dark matter fermion density. The construction of models that would yield the requisite self-interacting dark matter is left as a problem for the future.

If the neutron decays to multiple dark states, e.g., $n \rightarrow \chi+\phi$, where $\phi$ is a dark boson, our results hold when the $\phi$ do not carry a conserved charge and, thus, their chemical potential $\mu_{\phi}$ must vanish; if $\phi$ is a dark photon $\gamma_{D}$, for instance, obtaining $2 M_{\odot}$ neutron stars would still require $\chi$ 's to have strong repulsive self-interactions. Requiring the dark states to carry a conserved charge, and thus be part of a multiparticle dark sector, would allow $\mu_{\phi} \neq 0$ and possibly permit a resolution of the neutron lifetime puzzle as well as the construction of dark matter equations of state consistent with $2 M_{\odot}$ neutron stars. Such a scenario, an alternative to the dark matter sector having strong repulsive interactions, would also require some nonminimal multistate dark sector.

Beyond the immediate motivation provided by the neutron lifetime puzzle, the present study demonstrates that neutron stars are powerful probes of baryon-violating $n-\chi$ couplings for $\chi$ 's as heavy as $2 \mathrm{GeV}$. We stress that the present analysis considers only baryon-numberviolating couplings, in contrast to analyses of dark matter capture by neutron stars which focus on elastic scattering of dark matter on baryons [26-38]. The class of interactions we consider here can easily have elastic baryon- $\chi$ cross sections orders of magnitude below what can be tested with gravitational capture; in the regime $m_{\chi} \lesssim m_{n}$ relevant for the neutron lifetime puzzle, the bottle measurements stringently constrain the relevant coupling (implying $\sigma_{n \chi} \sim 10^{-54} \mathrm{~cm}^{2}$ ). Indeed, our results apply for all couplings large enough for baryon- $\chi$ conversion to reach equilibrium within the neutron star; even conversion times of years, many orders of magnitude longer than that required to address the neutron lifetime puzzle, would lead to equilibrium.

Nonzero strangeness in the quark matter phase in the interior of neutron stars would also allow analogous tests of a baryon-number-violating coupling of $\chi$ to hyperons. As strange baryons are far less abundant in nature than neutrons, a $\chi$-hyperon coupling would be far more challenging to test in the lab, making neutron stars even more valuable probes.

As we have shown, neutron stars can be used to constrain dark matter models in ways that are simply inaccessible to other probes, whether cosmological or terrestrial, and thus provide a vital new window onto $\mathrm{GeV}$-scale dark sectors. 
This research was supported in part by National Science Foundation Grants No. PHY-1506416 and No. PHY1714042, and Department of Energy Early Career Grant No. DE-SC0017840.

Note added.-Recently, several related works have appeared. Notably, Refs. [39,40] reach very similar conclusions to our own, while Ref. [41] describes a search for neutron decays into $\chi+e^{+} e^{-}$, with negative results, and Ref. [42] builds an equation of state for self-interacting dark matter within a neutron star.

[1] A. T. Yue, M. S. Dewey, D. M. Gilliam, G. L. Greene, A. B. Laptev, J. S. Nico, W. M. Snow, and F. E. Wietfeldt, Phys. Rev. Lett. 111, 222501 (2013).

[2] J. Byrne and P. G. Dawber, Europhys. Lett. 33, 187 (1996).

[3] A. P. Serebrov et al., Phys. Rev. C 97, 055503 (2018).

[4] R. W. Pattie, Jr. et al., Science 360, 627 (2018).

[5] S. Arzumanov, L. Bondarenko, S. Chernyavsky, P. Geltenbort, V. Morozov, V. V. Nesvizhevsky, Y. Panin, and A. Strepetov, Phys. Lett. B 745, 79 (2015).

[6] A. Steyerl, J. M. Pendlebury, C. Kaufman, S. S. Malik, and A. M. Desai, Phys. Rev. C 85, 065503 (2012).

[7] A. Pichlmaier, V. Varlamov, K. Schreckenbach, and P. Geltenbort, Phys. Lett. B 693, 221 (2010).

[8] A. Serebrov et al., Phys. Lett. B 605, 72 (2005).

[9] W. Mampe, L. N. Bondarenko, V. I. Morozov, Y. N. Panin, and A. I. Fomin, Pis'ma Zh. Eksp. Teor. Fiz. 57, 77 (1993) [JETP Lett. 57, 82 (1993)].

[10] F. E. Wietfeldt and G. L. Greene, Rev. Mod. Phys. 83, 1173 (2011).

[11] G. L. Greene and P. Geltenbort, Sci. Am. 314, 36 (2016).

[12] C. Patrignani et al. (Particle Data Group), Chin. Phys. C 40, 100001 (2016).

[13] B. Fornal and B. Grinstein, Phys. Rev. Lett. 120, 191801 (2018).

[14] Z. Tang et al., Phys. Rev. Lett. 121, 022505 (2018).

[15] A. Czarnecki, W. J. Marciano, and A. Sirlin, Phys. Rev. Lett. 120, 202002 (2018).

[16] F. Özel and P. Freire, Annu. Rev. Astron. Astrophys. 54, 401 (2016).

[17] P. Demorest, T. Pennucci, S. Ransom, M. Roberts, and J. Hessels, Nature (London) 467, 1081 (2010).
[18] J. J. Antoniadis et al., Science 340, 1233232 (2013).

[19] The ATNF Pulsar Catalog, https://www.atnf.csiro.au/ people/pulsar/psrcat.

[20] G. Baym, T. Hatsuda, T. Kojo, P. D. Powell, Y. Song, and T. Takatsuka, Rep. Prog. Phys. 81, 056902 (2018).

[21] A. Akmal, V. R. Pandharipande, and D. G. Ravenhall, Phys. Rev. C 58, 1804 (1998).

[22] B. P. Abbott et al. (LIGO Scientific Collaboration), arXiv: 1805.11581.

[23] R. C. Tolman, Phys. Rev. 55, 364 (1939).

[24] J. R. Oppenheimer and G. M. Volkoff, Phys. Rev. 55, 374 (1939).

[25] S. W. Randall, M. Markevitch, D. Clowe, A. H. Gonzalez, and M. Bradac, Astrophys. J. 679, 1173 (2008).

[26] I. Goldman and S. Nussinov, Phys. Rev. D 40, 3221 (1989).

[27] C. Kouvaris, Phys. Rev. D 77, 023006 (2008).

[28] G. Bertone and M. Fairbairn, Phys. Rev. D 77, 043515 (2008).

[29] A. de Lavallaz and M. Fairbairn, Phys. Rev. D 81, 123521 (2010).

[30] C. Kouvaris and P. Tinyakov, Phys. Rev. D 82, 063531 (2010).

[31] S. D. McDermott, H. B. Yu, and K. M. Zurek, Phys. Rev. D 85, 023519 (2012).

[32] T. Güver, A. E. Erkoca, M. Hall Reno, and I. Sarcevic, J. Cosmol. Astropart. Phys. 05 (2014) 013.

[33] J. Bramante, K. Fukushima, and J. Kumar, Phys. Rev. D 87, 055012 (2013).

[34] N. F. Bell, A. Melatos, and K. Petraki, Phys. Rev. D 87, 123507 (2013).

[35] B. Bertoni, A. E. Nelson, and S. Reddy, Phys. Rev. D 88, 123505 (2013).

[36] J. Bramante, K. Fukushima, J. Kumar, and E. Stopnitzky, Phys. Rev. D 89, 015010 (2014).

[37] M. Baryakhtar, J. Bramante, S. W. Li, T. Linden, and N. Raj, Phys. Rev. Lett. 119, 131801 (2017).

[38] N. Raj, P. Tanedo, and H. B. Yu, Phys. Rev. D 97, 043006 (2018).

[39] D. McKeen, A. E. Nelson, S. Reddy, and D. Zhou, following Letter, Phys. Rev. Lett. 121, 061802 (2018).

[40] T. F. Motta, P. A. M. Guichon, and A. W. Thomas, J. Phys. G 45, 05LT01 (2018).

[41] X. Sun et al., Phys. Rev. C 97, 052501 (2018).

[42] J. M. Cline and J. M. Cornell, arXiv:1803.04961. 